\title{
The new approach to identification of film reflecting holographic marks
}

\author{
S.O. Kostyukevych ${ }^{a}$, L.I. Muravsky ${ }^{b^{*}}$, V.M. Fitio ${ }^{c}$, T.I. Voronyak ${ }^{b}$, \\ P.E. Shepeliavyi ${ }^{a}$, K.V. Kostyukevych ${ }^{a}$, N.L. Moskalenko ${ }^{a}$, V.I. Pogoda ${ }^{a}$ \\ ${ }^{a}$ V.Lashkaryov Inst. of Semicond. Physics of the Nat. Acad. Sci. of Ukraine, Kyiv 03028, Ukraine \\ ${ }^{b}$ Karpenko Physico-Mechanical Inst. of the Nat. Acad. Sci. of Ukraine, Lviv 79601, Ukraine \\ "National University "Lvivska Polytechnica”, Lviv 79013, Ukraine \\ *dep24@ah.ipm.lviv.ua; phone +380322-633388; fax +380 322-649427
}

\begin{abstract}
The new approach for creation of film reflecting holographic marks for optical security is proposed. Such marks are replicas of a reflecting master hologram recorded on a chalcogenide glass layer. To receive the master hologram, the joint power spectrum of a reference phase mask and an input phase mask or a transformed phase mask is produced at the plane of the hologram writing and is modulated by an inclined laser beam. If an inclined laser beam illuminates the replica recorded on a flexible substrate, phase noise including speckle noise is eliminated because the hologram carrier frequency exceeds greatly the limiting frequency of the phase noise power spectrum. Experimental results have shown the principal possibility to produce the high performance film reflecting holographic marks for security applications. The proposed approach can be combined with technologies for fabrication of rainbow holograms. In this case, the received rainbow holograms are the reflecting holographic marks simultaneously. Therefore, they can be verified not only visually, but also by means of automatic identification.
\end{abstract}

Keywords: reflecting holographic mark, chalcogenide glass layer, joint transform correlator, phase mask, transformed phase mask, optimum exposure, correlation peak, identification, security verification.

Manuscript received 31.01.11; accepted for publication 14.09.11; published online 21.09.11

\section{Introduction}

Recently, identification and security verification of different products, things and documents by optical methods and means are developed intensively. ${ }^{1-3}$ The optical methods and systems possess large possibilities to resist the counterfeiting due to the parallel encodingdecoding of large amount of information and, in particular, 2D and 3D arrays. The techniques utilizing random phase masks simultaneously with fingerprints, ${ }^{2-7}$ in particular, the security verification technique ${ }^{2,4}$ and the method for optical protection and identification of documents, ${ }^{6,7}$ are very perspective among optical security technologies.

Mentioned above techniques ensure reliable protection only of such objects, which can contain random phase masks frequently combined with fingerprints. Meanwhile, the majority of hardware products and things are not suitable for protection by transparent optical marks. One of possible solutions of this problem is the creation of a reflecting optical mark by recording a joint power spectrum (JPS) of a transformed and reference phase masks on a chalcogenide glass $\mathrm{As}_{40} \mathrm{~S}_{40} \mathrm{Se}_{20}$ at the first stage of a joint transform correlator (JTC) scheme. ${ }^{8}$ The resulting reflecting optical mark is fabricated as a copy of the written JPS. However, reflecting optical marks can be copied only on a solid substrate. So, only solid surfaces of objects to be protected from counterfeiting can be used for bonding of such marks. If the written JPS is copied on a film substrate, the optical beam reflecting from its surface does not reconstruct satisfactorily the correlation peaks in the first diffraction orders of the JTC output plane due to influence of power spectrum density of phase noise (including speckle noise) caused by any microdeformation of a film layer and its optically 


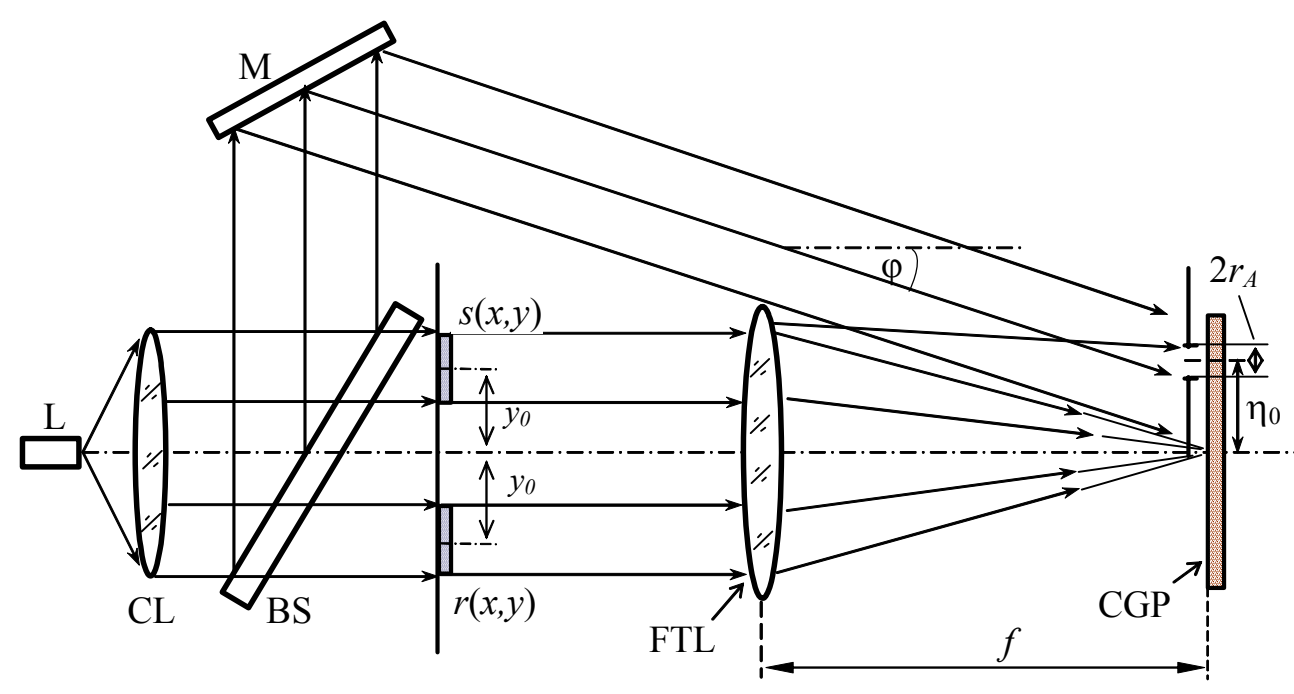

Fig. 1. Optical arrangement for a master hologram writing on a chalcogenide glass layers $\mathrm{As}_{40} \mathrm{~S}_{60-\mathrm{x}} \mathrm{Se}_{\mathrm{x}}$ : $\mathrm{L}-\mathrm{He}-\mathrm{Cd}$ laser $\Lambda \Gamma-70$; CL - collimating lens; BS - beam splitter; M - mirror; FTL - Fourier transform lens; CGP - chalcogenide glass plate.

rough surface. The phase noise power spectrum density is band limited. For example, the highest spatial frequency of the wideband speckle noise power spectrum density is equal to resolution limit of an aberration-free Fourier lens ${ }^{9}$ used in the JTC first stage. To separate the phase noise power spectrum from a JPS of an input and a random phase mask, we propose to record a reflecting Fourier hologram on a chalcogenide glass $\mathrm{As}_{40} \mathrm{~S}_{60-\mathrm{x}} \mathrm{Se}_{\mathrm{x}}$ layer, where $\mathrm{x}=0,10,20$. Such hologram can be considered as a master hologram used for replication of film reflecting holographic marks (RHMs). Each RHM is suitable for reconstruction of correlation peaks, if it is illuminated by an inclined laser beam.

\section{Conditions of master hologram recording and reconstruction}

As shown in our previous paper $^{8}$, procedures of a reflecting optical mark fabrication and its identification can be realized on the basis of a JTC architecture. The first stage of this correlator was used to produce a reflecting optical mark written on a solid substrate, for example, on a chalcogenide glass $\mathrm{As}_{40} \mathrm{~S}_{40} \mathrm{Se}_{20}$ layer. In linear approximation, this mark can be considered as a JPS of an input transformed phase mask and a reference phase mask. The JTC second stage was used for identification of this mark. Our approach is differed from previous only by using the inclined reference parallel laser beam to produce the reflecting master hologram. The carrier frequency of this beam should exceed greatly the upper frequency of a phase noise power spectrum. Both a phase mask and a transformed phase mask can be used as an input mask to produce a master hologram. The arrangement for recording of a master hologram is shown in Fig. 1. To identify the RHM replicated on a flexible substrate, the parallel laser beam illuminates the RHM surface. If the slope angle of this beam is identical to the slope angle of the writing beam, the desired spectral response containing Fourier spectra of an input and reference masks is reconstructed along an optical axis normal to the plane of the RHM placing. If a phase mask is used as an input mask, the identification criteria are based only on the intensity of one correlation peak. ${ }^{4,5}$ If a transformed phase mask is used as an input mask, the identification criteria became considerably larger than in the first case, because the quantity of output correlation peaks is equal to quantity of separate fragments of the transformed phase mask. In this case, not only peak intensities, but also vector distances between peaks can be regarded as identification criteria. ${ }^{6,7}$ Let us consider briefly the linear and nonlinear models of such the RHM's producing and identification.

\subsection{Linear model.}

To simplify calculations, we consider only the deterministic model of a binary phase mask. Let the rectangular binary phase mask consists of $M \times N$ identical rectangular pixels $a \times b$ and the mask size is equal $M a \times N b$. Then the complex amplitude transmittance of each pixel can be expressed as

$t_{m, n}(x, y)=\operatorname{rect}\left(\frac{x-x_{m}}{a}, \frac{y-y_{n}}{b}\right)$,
$\exp \left[j \theta\left(x-x_{m}, y-y_{n}\right)\right]$

where $x_{m}=c m-\frac{c}{2}(M+1), \quad y_{n}=-d n+\frac{d}{2}(N+1)$ are

the coordinates of the pixel center; $m=1,2, \ldots, M$; $n=1,2, \ldots, N ; \theta$ is the pixel phase equal to $2 \pi l$ or $2 \pi l+\pi, l=0,1,2, \ldots$.

\section{(C) 2011, V. Lashkaryov Institute of Semiconductor Physics, National Academy of Sciences of Ukraine}


Let a reference phase mask $r$ and an input phase mask $s$ are entered into an input plane $(x, y)$ of a conventional JTC (see Fig. 1). If these masks are identical, they can be expressed as ${ }^{7,8}$

$$
\begin{aligned}
r(x, y) & \sum_{m=1}^{M} \sum_{n=1}^{N}\left[\operatorname{rect}\left(\frac{x-x_{m+}}{a}, \frac{y-y_{n+}+y_{0}}{b}\right)-\right. \\
- & \left.\operatorname{rect}\left(\frac{x-x_{m-}}{a}, \frac{y-y_{n-}+y_{0}}{b}\right)\right], \\
s(x, y)= & \sum_{m=1}^{M} \sum_{n=1}^{N}\left[\operatorname{rect}\left(\frac{x-x_{m+}}{a}, \frac{y-y_{n+}-y_{0}}{b}\right)-\right. \\
& \left.-\operatorname{rect}\left(\frac{x-x_{m-}}{a}, \frac{y-y_{n-}-y_{0}}{b}\right)\right],
\end{aligned}
$$

where $x_{m+}, y_{n+}$ are the coordinates of the pixel with the complex transmittance $t_{m, n}=+1 ; x_{m-}, y_{n-}$ are the coordinates of the pixel with the complex transmittance $t_{m, n}=-1 ; 2 y_{0}$ is the distance between two masks. Let us the inclined parallel laser beam illuminating the chalcogenide glass plate disposed in the JTC frequency domain is expressed by next equation:

$H(v)=A_{0} \exp \left(-j 2 \pi y_{h} v\right)$,

where $v$ is the spatial frequency along the axis $\vec{\eta}$ in the Fourier plane, $y_{h}=f \cdot \operatorname{tg} \varphi, \varphi$ is the angle of the parallel laser beam inclination to the optical axis of the JTC first stage, $f$ is the focal length of a Fourier transform lens (see Fig. 1). If a reflecting photosensitive material disposed in the JTC Fourier plane is linear and the complex amplitude transmittances of input and reference phase masks are identical, i.e. $r\left(x, y-y_{0}\right)=s\left(x, y+y_{0}\right)$, the intensity distribution of the reconstructed interference pattern recorded on this material is proportional to

$$
\begin{aligned}
& |T(u, v)|^{2}=\left|R(u, v)+S(u, v)+A_{0} \exp \left(-j 2 \pi y_{h} v\right)\right|^{2}= \\
& R R^{*}+S S^{*}+\left|A_{0}\right|^{2}+R S^{*}+R * S+ \\
& +\left[R A_{0}^{*} \exp \left(j 2 \pi y_{h} v\right)+S A_{0}^{*} \exp \left(j 2 \pi y_{h} v\right)\right]+ \\
& {\left[R^{*} A_{0} \exp \left(-j 2 \pi y_{h} v\right)+S^{*} A_{0} \exp \left(-j 2 \pi y_{h} v\right)\right],}
\end{aligned}
$$

where $*$ is the complex conjugation symbol,

$$
\begin{aligned}
& R(u, v)=F[r(x, y)]= \\
& =(a b) \sin c(a u) \sin c(b v) \exp \left(\mathrm{j} 2 \pi y_{0} v\right) W_{m, n}(u, v), \\
& S(u, v)=F[s(x, y)]=(a b) \sin c(a u) \sin c(b v) \times \\
& \times \exp \left(-\mathrm{j} 2 \pi y_{0} v\right) W_{m, n}(u, v), \\
& W_{m, n}(u, v)=\sum_{m=1}^{M} \sum_{n=1}^{N}\left[\exp \left(-j 2 \pi x_{m+} u\right) \exp \left(-j 2 \pi y_{n+} v\right)-\right.
\end{aligned}
$$

$F$ is the symbol of 2 D Fourier transform.
If the same parallel laser beam expressed by Eq. (4) illuminates a master hologram recorded on a photosensitive material, the signal reconstructed along the optical axis of the JTC second stage can be expressed as

$$
\begin{aligned}
& U(u, v)=A_{0} \exp \left(-j 2 \pi y_{h} v\right) \\
& {\left[R A_{0}^{*} \exp \left(j 2 \pi y_{h} v\right)+S A_{0}^{*} \exp \left(j 2 \pi y_{h} v\right)\right]=\left|A_{0}\right|^{2}(R+S) .}
\end{aligned}
$$

This signal is produced by reconstruction of the sixth term $\left[R A_{0}^{*} \exp \left(j 2 \pi y_{h} v\right)+S A_{0}^{*} \exp \left(j 2 \pi y_{h} v\right)\right]$ of the intensity distribution $|T(u, v)|^{2}$ expressed by Eq. (5). Signal $U(u, v)$ is projected on an optically addressed SLM or a digital camera by using the telecentric imaging system containing two lenses. In this imaging system, the front focal plane of the first lens should coincide with the disposition plane of a sensitive material surface and the back focal plane of the second lens should coincide with the plane of a SLM or a sensor array disposition. The output correlation field can be obtained by Fourier transform of the recorded by a linear optically addressed SLM or a digital camera intensity distribution proportional to $|U(u, v)|^{2}$, which is expressed as

$|U(u, v)|^{2}=\left|A_{0}\right|^{4}\left(R R^{*}+S S *+R S^{*}+R * S\right)$.

The desired output correlation field is produced after optical Fourier transform of intensity distribution $|U(u, v)|_{M}^{2}$ recorded on a SLM or after digital Fourier transform of intensity distribution $|U(u, v)|_{D}^{2}$ recorded by a digital camera. If the image sampling is not taken in account, the linear approximation of complex amplitude spatial distribution of both $|U(u, v)|_{M}^{2}$ and $|U(u, v)|_{D}^{2}$ Fourier transforms can be written as $C\left(x^{\prime}, y^{\prime}\right)=C_{0}\left(x^{\prime}, y^{\prime}\right)+C_{+}\left(x^{\prime}, y^{\prime}\right)+C_{-}\left(x^{\prime}, y^{\prime}\right)=$ $=(M N)(a b)\left[2 \Lambda\left(\frac{x^{\prime}}{a}, \frac{y^{\prime}}{b}\right)+\Lambda\left(\frac{x^{\prime}}{a}, \frac{y^{\prime}-2 y_{0}^{\prime}}{b}\right)+\right.$ $\left.\Lambda\left(\frac{-x^{\prime}}{a}, \frac{-y^{\prime}+2 y_{0}^{\prime}}{b}\right)\right]+2 \widetilde{n}_{0}\left(x^{\prime}, y^{\prime}\right)+\widetilde{n}\left(x^{\prime}, y^{\prime}\right)+$ $\tilde{n}\left(-x^{\prime},-y^{\prime}\right)$,

where $C_{0}\left(x^{\prime}, y^{\prime}\right)$ is the signal located in the $\mathrm{dc}$; $C_{+}\left(x^{\prime}, y^{\prime}\right), C_{-}\left(x^{\prime}, y^{\prime}\right)$ are the correlation signals located in the +first and -first diffraction orders respectively; $2(M N)(a b) \Lambda\left(\frac{x^{\prime}}{a}, \frac{y^{\prime}}{b}\right)$ is the autocorrelation peak amplitude distribution in the $\mathrm{dc}$; $(M N)(a b) \Lambda\left(\frac{ \pm x^{\prime}}{a}, \frac{ \pm y^{\prime} \mp 2 y_{0}^{\prime}}{b}\right)$ is the correlation peak amplitude distribution in the \pm first order; $\tilde{n}_{0}\left(x^{\prime}, y^{\prime}\right)$, $\tilde{n}\left(x^{\prime}, y^{\prime}\right)$ and $\tilde{n}\left(-x^{\prime},-y^{\prime}\right)$ are the cross-talk noise 


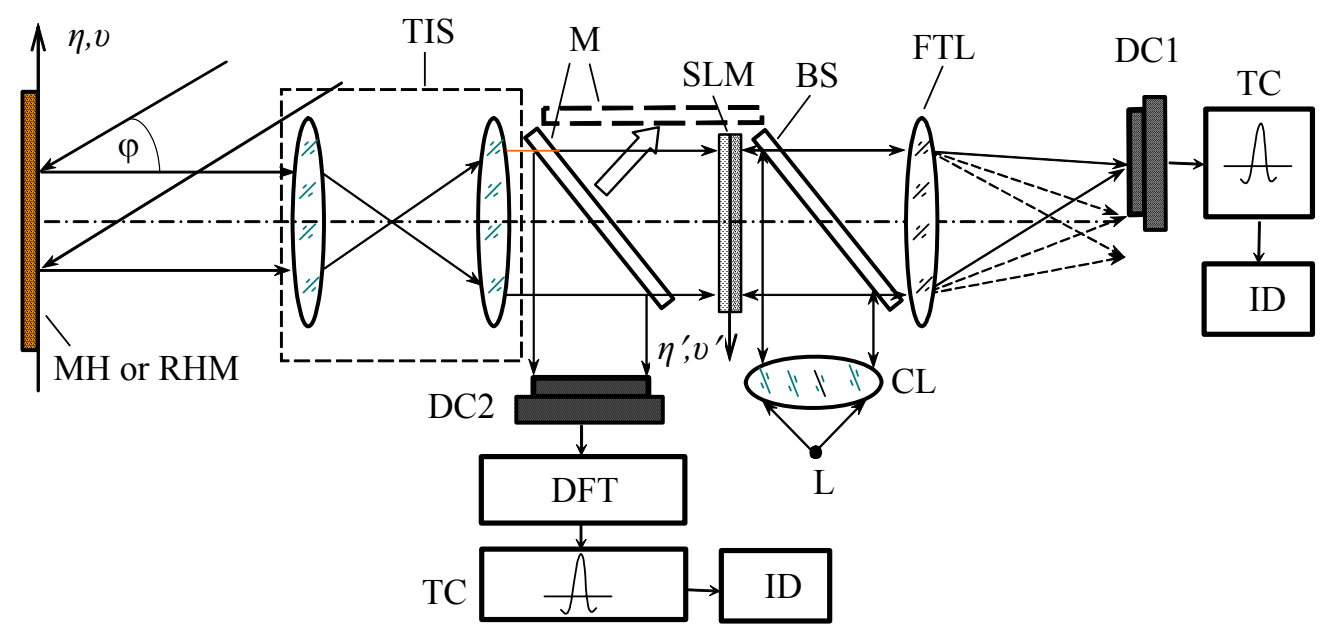

Fig. 2. Arrangement of a master hologram or a replicated RHM reconstruction: MH - master hologram; TIS - telecentric imaging system; M - mirror; SLM - optically addressed spatial light modulator; L - laser; CL - collimating lens; BS - beamsplitter; FTLFourier transform lens; DC1, DC2 - digital cameras; DFT - digital Fourier transform; TC - threshold comparison; ID identification device; $\eta, v$, axes of planes $(\xi, \eta)$ and $(u, v) ; \eta^{\prime}, v^{\prime}$, axes of planes $\left(\xi^{\prime}, \eta^{\prime}\right)$ and $\left(u^{\prime}, v^{\prime}\right)$.

amplitude distributions in the dc, + first and - first orders respectively. The arrangement of reconstruction of a master hologram or a replicated RHM is shown in Fig. 2.

\subsection{Nonlinear model.}

We have proposed one of possible approaches for evaluation of output response in a nonlinear JTC with photosensitive materials in the frequency domain, if the reference and input phase masks are entered into the JTC input plane. ${ }^{8,10}$ This approach is based on fulfillment of two basic conditions: i) definition of the diffraction efficiency $\eta$ versus exposure $E$ experimental dependence (exposure characteristic) for a photosensitive medium or a SLM; ii) mathematical representation of the interference pattern, which should be written on a photosensitive medium or a SLM. If the exposure characteristic and the function describing or approximating the written interference pattern are known, one can find the optimum writing exposure $E_{o p t}$, the reading round aperture's $A_{r}$ normalized radius $\rho_{A}=\sqrt{\left[a\left(u-u_{A}\right)\right]^{2}+\left[b\left(v-v_{A}\right)\right]^{2}}$ and the normalized distance $w_{A}=\sqrt{\left(a u_{A}\right)^{2}+\left(b v_{A}\right)^{2}}$ from the frequency plane $(u, v)$ origin to the aperture center, where $u_{A}, v_{A}$ are the coordinates of the aperture center. First condition for realization of the proposed approach can be satisfied experimentally. For this purpose, we can measure the exposure characteristic $\eta=\eta(E)$ of a chalcogenide glass layer $\mathrm{As}_{40} \mathrm{~S}_{50} \mathrm{Se}_{10}$ by using the conventional optical scheme for writing of reflecting holographic gratings. To satisfy second condition, it is necessary to provide the computer simulation of Eq. (5). But both the computer simulations of Eq. (5) and further procedures of its transformation demand the program design and the large amount of computations. Therefore, we have decided to determine $E_{o p t}, \rho_{A}$ and $w_{A}$ during experimental recording of RHMs and their reconstruction.

\section{Experimental results}

The application of a chalcogenide glass as an inorganic photoresist with high resolution $\left(\sim 5 \cdot 10^{3} \mathrm{~mm}^{-1}\right)$ is based on the effect of photostimulated change of chalcogenide glass film solubility in its selective solvent. ${ }^{11}$ Such films are produced by thermal vacuum film deposition. The mentioned above effect is exhibited in increase or decrease of the dissolution rate of irradiated sections in comparison with the dissolution rate of a non-irradiated layer depends upon the solvent type. In these cases, the positive or negative selective etching of a chalcogenide glass layer is occurred. Such photoresists are successfully used for making of holographic diffraction elements, synthesized holograms, konoforms, master mirror disks (CD, DVD) etc. ${ }^{12}$

In order to produce the master holograms, we have used chalcogenide glass layers $\mathrm{As}_{40} \mathrm{~S}_{60-\mathrm{x}} \mathrm{Se}_{\mathrm{x}}$, where $\mathrm{x}=0$; 10. To fabricate the plates containing such layers, firstly a chromium (Cr) layer with thickness equal to $30 \mathrm{~nm}$ was coated on a glass substrate, secondly a chalcogenide glass layer $\mathrm{As}_{40} \mathrm{~S}_{60-\mathrm{x}} \mathrm{Se}_{\mathrm{x}}$ with thickness equal to $\sim 500 \mathrm{~nm}$ was coated on a Cr layer. The coating was realized by step-bystep thermal spraying in vacuum with the residual pressure $<10^{-3}$. The etching of an exposed chalcogenide glass layer was realized in an anhydrous organic selective etchant of negative type. ${ }^{13}$ The plate can contain only a chalcogenide glass layer. But if the $\mathrm{Cr}$ layer is evaporated previously on a glass substrate, the diffraction efficiency of such hologram can be increased greatly. Besides, the hologram wear resistance is increased significantly. 


\subsection{Measurement of diffraction efficiency of a chalcogenide glass layer}

For approximate estimation of optimum exposure $E_{\text {opt }}$, we have measured the exposure characteristic $\eta=\eta(E)$ of a chalcogenide glass layer $\mathrm{As}_{40} \mathrm{~S}_{50} \mathrm{Se}_{10}$. This characteristic was measured by using the conventional optical arrangement for writing of reflecting holographic gratings. A He-Cd laser $\left(\lambda_{w}=442 \mathrm{~nm}\right)$ was used for their writing with different exposures. The reference beam angle was equal to $30 \mathrm{deg}$ concerning the optical axis of this arrangement. The diffraction efficiency of each written grating was measured by using the usual arrangement of a reflecting grating reading by a laser diode module MLRZ-650-005 $\left(\lambda_{r}=650 \mathrm{~nm}\right)$. The received dependence $\eta=\eta(E)$ is depicted in Fig. 3. Calculations of the optimum exposure represented in our previous paper ${ }^{8}$ have shown that one is disposed on the dip of curve $\eta=\eta(E)$ after reaching its peak value, if 0.1 $\leq \rho_{A} \leq 0.4$ and $w_{A} \leq 0.66$. So, we chose the exposures in the range from $100 \mathrm{~mJ} / \mathrm{cm}^{2}$ up to $200 \mathrm{~mJ} / \mathrm{cm}^{2}$ and the normalized radius $\rho_{A}=0.1$ disposed on the normalized distance $w_{A}=0.5$ from the frequency plane $(u, v)$ origin.

\subsection{Writing of reflecting master holograms and replication of RHMS}

The binary reference and transformed masks were used for master hologram writing on a chalcogenide glass layers $\mathrm{As}_{40} \mathrm{~S}_{60-\mathrm{x}} \mathrm{Se}_{\mathrm{x}}$. Each the mask contains $256 \times 256$ adjacent square pixels with complex transmittances equal to +1 or -1 for wavelength $\lambda=442 \mathrm{~nm}$. In these masks, the dimensions of pixels are equal to $20 \times 20 \mu \mathrm{m}$ and the pixel pitch is equal to $20 \mu \mathrm{m}$. The transformed phase masks consisting of two, three and nine fragments of a reference phase mask were used together with a reference phase mask to produce a JPS. The configuration of the two-fragment transformed mask made from the reference phase mask is shown in Fig. 4.

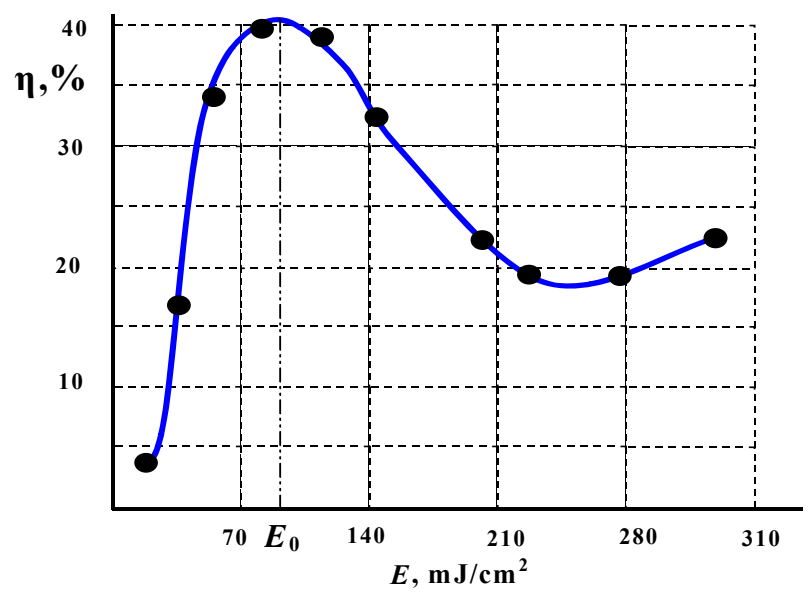

Fig. 3. Dependence $\eta=\eta(E)$ for a chalcogenide glass $\mathrm{As}_{40} \mathrm{~S}_{50} \mathrm{Se}_{10}$.

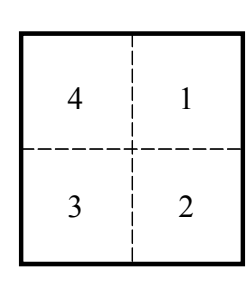

a)

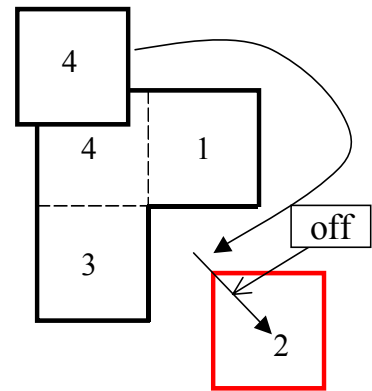

b)

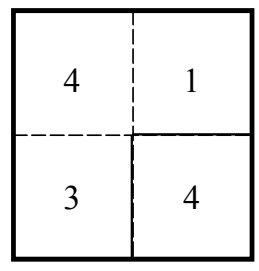

c)

Fig. 4. Procedure of the first stage of the two-fragment transformed phase mask fabrication: reference phase mask (a); separation of reference mask on two fragments: replication of quadrant 4 and replacement of quadrant 2 by quadrant 4 (b); transformed phase mask (c).

The optical arrangement for the master hologram writing on a chalcogenide glass layers $\mathrm{As}_{40} \mathrm{~S}_{60-\mathrm{x}} \mathrm{Se}_{\mathrm{x}}$ is represented in Fig. 1. This arrangement containing a Fourier lens with focal length $f=700 \mathrm{~mm}$ is based on a JTC first stage. The binary reference and transformed masks were entered and the distance between two masks was equal to $8.0 \mathrm{~mm}$. The collimated parallel $\mathrm{He}-\mathrm{Cd}$ laser $\left(\Lambda \Gamma-70, \lambda_{w}=442 \mathrm{~nm}\right)$ beam was used for illumination of phase masks and shaping of a reference parallel laser beam illuminated the chalcogenide glass layer at angle $\varphi=30 \mathrm{deg}$ concerning the optical axis of the arrangement. To adjust the intensities of a JPS and a reference beam and estimate the desired range of exposures, first we entered the calibrated photodiode $\Phi \mathrm{D}-7 \mathrm{~K}$ in the frequency plane $(u, v)$ and disposed its center on the distance $\eta_{0}=\frac{\pi}{b} w_{A, v} \lambda_{w} f=24.3 \mathrm{~mm}$ from the frequency plane origin. The distance $\eta_{0}$ corresponds to normalized distance $w_{A, v}=b v=0.5$ along the axis $\vec{v}$, which evaluates the position of a center of a round aperture $A_{r}$ with normalized radius $\rho_{A}=0.1$. Because the radius of this aperture is equal to $r_{A}=\frac{\pi}{b} \rho_{A} \lambda_{w} f=4.86$ $\mathrm{mm}$, we have mounted the round aperture with diameter $2 r_{A}=9.7 \mathrm{~mm}$ directly in front of the photodiode along the direction opposite to the direction of the reference beam propagation (see Fig. 1). The measured mean intensity $I_{m}(u, v)$ of the JPS part selected by the round aperture was equal to $0.8 \mathrm{~mW} / \mathrm{cm}^{2}$. To ensure maximum 
modulation of a selected JPS area, we have chosen the same intensity of the reference beam measured by this photodiode at the same place. So, the exposure time range for writing of reflecting master holograms on a chalcogenide glass layer was calculated by using the justified above exposure range and measured mean intensities of the JPS and the reference light beam. This time was defined over the range from $62 \mathrm{~s}$ up to $125 \mathrm{~s}$. Because the area for writing of a master hologram is restricted by a round aperture, we have recorded several master holograms on the same chalcogenide glass layer.

To fabricate RHMs, we have used the photopolymer "Yantar'-3" developed in the Institute of Macromolecular chemistry of the NAS of Ukraine. All master holograms written on chalcogenide glass layers $\mathrm{As}_{40} \mathrm{~S}_{50} \mathrm{Se}_{10}$ and $\mathrm{As}_{40} \mathrm{~S}_{60}$ were replicated on this photopolymer. The photopolymer was deposited on a lavsan carrier. After replication of a master hologram on a photopolymer, the photopolymer surface was coated by a thin Al layer.

\subsection{Reconstruction and analysis of RHMs and refelecting master holograms}

The experimental setup arrangement for reconstruction of RHMs and reflecting master holograms is shown in Fig. 5. The optical part of this setup contains a laser diode module MLRZ-650-005 to produce an expanded parallel laser beam $\left(\lambda_{r}=650 \mathrm{~nm}\right)$ illuminating a RHM or a master hologram at the same angle of $30 \mathrm{deg}$. The reconstructed response $U(u, v)$ evaluated by Eq. (6) in linear approximation is projected by a telecentric imaging system containing two lenses with focal lengths $f_{1}$ and $f_{2}$ on a CCD camera "FINE" T-392C. The telecentric imaging system is placed along the optical axis normal to the plane of a reflecting hologram disposition. The horizontal camera resolution is equal to $81.6 \mathrm{lp} / \mathrm{mm}$ and the vertical camera resolution is equal to $58 \mathrm{lp} / \mathrm{mm}$, the sensor array's active (image) area is equal to $4.9 \mathrm{~mm}$ by $3.7 \mathrm{~mm}$. To ensure the reliable reconstruction of a JPS recorded by the CCD camera and evaluated by Eq. (7), we selected the lenses in the telecentric imaging system, which focal lengths $f_{1}$ and $f_{2}$ are related as $2: 1$. In this case, the JPS carrier spatial frequency that is equal to $26 \mathrm{~mm}^{-1}$ is translated to $52 \mathrm{~mm}^{-1}$ and the carrier spatial frequency of a recorded JPS does not exceed the vertical camera resolution. Such demagnification of the telecentric system allows recording all area of an interference pattern projected on the sensor array from a chalcogenide glass layer in horizontal direction and only three fourth of this area in vertical direction. The JPS recorded by the CCD camera is captured by a frame grabber and entered into a PC. Due to the Digital Fourier transform of the digitized JPS, the output correlation response containing several correlation peaks in the \pm first diffraction order is produced. The part of correlation response covering the first diffraction order area is used for creation of an identification feature vector and its comparison with a reference feature vector. 6

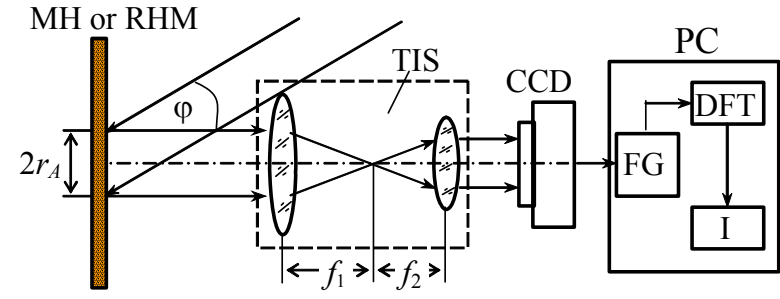

Fig. 5. Experimental setup arrangement for reconstruction of RHMs and refelecting master holograms: $\mathrm{MH}$, master hologram; RHM, reflecting holographic mark; TIS, telecentric imaging system; M, mirror; CCD, CCD camera; PC, personal computer; FG, frame grabber; DFT, digital Fourier transform; I, identification.

The results of reconstruction of master holograms and RHMs are represented in Fig. 6 and Fig. 7 respectively. In this case, the two-fragment transformed phase mask represented in Fig. 4, was used together with the reference phase mask for writing the master hologram. These Figures show that two peaks in \pm first diffraction order are very sharp and narrow. In Fig. 6, the peak at the center of the \pm first diffraction order is produced as a result of autocorrelation of the transformed mask larger fragment with the corresponding fragment of the reference mask, and the second peak is the product of autocorrelation of the transformed mask smaller fragment with fourth quadrant of the reference mask. In Fig. 7, the correlation peaks are dissymmetrical to the correlation peaks reperesented in Fig. 6. Such behavior of the correlation peaks is clear, because all studied RHMs are mirror reflections of corresponding master holograms. The produced peaks can be identified easily by automatic means.

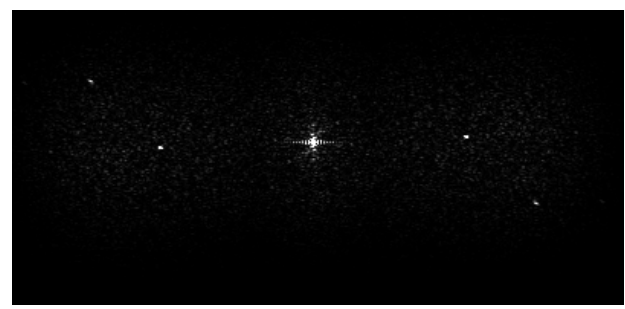

Fig. 6. Correlation peaks in + first and -first diffraction orders as a result of reconstruction of the master hologram. Twofragment transformed phase mask (see Fig. 4) was used to record the master hologram.

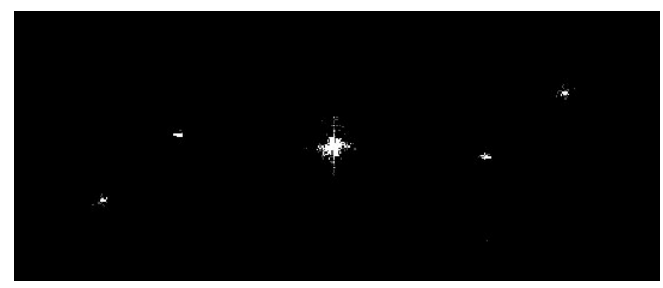

Fig. 7. Correlation peaks in +first and -first diffraction orders as a result of reconstruction of the RHM. Two-fragment transformed phase mask (see Fig. 4) was used to record the master hologram. 


\section{Conclusions}

The proposed approach for fabrication and identification of RHMs on a flexible substrate has some advantages in comparison with the previous similar approach for fabrication and identification of ROMs on a solid substrate. ${ }^{8}$ The main advantage consists in removal of the phase and speckle noise caused by any microdeformation of a film layer and its optically rough surface. This noise does not allow reconstructing the correlation response from ROM's replicas written on a flexible substrate. The fulfilled experiments with identification of RHMs have shown the high performance of photopolymer RHMs on a lavsan carrier. Such RHMs can be used successfully for identification and security verification of different products and things.

Because the proposed approach can be combined with technologies for fabrication of rainbow holograms, the unique possibility of quantitative identification and security verification of rainbow holograms fabricated by using our approach became realizable. Indeed, such rainbow holograms can be treated simultaneously as RHMs. Therefore they can be verified not only visually, but also by means of automatic identification. The created setup for reconstruction of RHMs can be used as a prototype of an automatic system for identification and security verification of rainbow holograms.

\section{References}

1. R.L. van Renesse., Ed., Optical Document Security, Second ed., Artech House, Norwood / London (1998).

2. B. Javidi, "Securing information with optical technologies," Phys. Today 50, 27-32, 1997.

3. "Special Section on Optical Security," J.L. Horner, B. Javidi, Eds., Opt. Eng. 38, 8-119, 1999.

4. B. Javidi, J.L. Horner, "Optical pattern recognition for validation and security verification," Opt. Eng. 33, 1752-1756, 1994.
5. E.G. Johnson, J.D.Brasher, D.A Gregory, P. Erbach, M. Duignan, G. Behrman, S.H. Lee, W. Daschner, P. Long, "Optical recognition of phaseencrypted biometrics," Opt. Eng. 37, 18-26, 1998.

6. L.I. Muravsky, T.I. Voronyak, V.M. Fitio, M.V. Shovgenyuk, "Transformed phase mask and photoanisotropic material in optical correlators applied for security verification," Opt. Eng. 38, 2532, 1999.

7. L.I. Muravsky, "Optical correlation systems for fingerprint and phase mask identification," A.A. Akaev, S.B. Gurevich, K.M. Jumaliev, L.I. Muravsky, T.A. Smirnova, Holography and optical information processing: selected sections, 227-444, Uchkun, Bishkek, St. Petersburg, 2003 (in Russian).

8. L.I. Muravsky, S.O. Kostyukevych, T.I. Voronyak, P.E. Shepeliavyi, "Study of chalcogenide glass based reflecting optical marks," SPIE-IS\&T Electronic Imaging Proc. 5310, 377-386, 2004.

9. I. Yamaguchi, "Fundamentals and applications of speckle," SPIE Proc. 4933, 1-8, 2003.

10. L.I. Muravsky, "Identification of random phase masks in a nonlinear joint transform correlator," SPIE Proc. 4148, 185-193, 2000.

11. B.T. Kolomietc, V.M. Lyubin, V.P. Shilo, "Photostimulated changes of chalcogenide glass solubility," Phys. And Chem. of Glass, 4, 351-357, 1978 (in Russian).

12. I.Z. Indutnyi , A.V. Stronski, S.A. Kostyukevych, P.F. Romanenko , P.E. Shepeliavyi, I.I. Robur, "Holographic optical elements fabrication using chalcogenide layers," Opt. Eng. 34, 1030-1039, 1995.

13. Patent of the Russian Federation \#2008285. I.Z. Indutnyi, S.A. Kostyukevych, P.E. Shepeliavyi, "Solution for negative etching of chalcogenide glasses," Otkrytiya, izobretieniya, \#4, 1994 (in Russian) 